\title{
On the isomorphism problem for even Artin groups
}

\author{
RUBÉN BLASCO-GARCÍA \\ LUIS PARIS
}

\begin{abstract}
An even Artin group is a group which has a presentation with relations of the form $(s t)^{n}=(t s)^{n}$ with $n \geq 1$. With a group $G$ we associate a Lie $\mathbb{Z}$-algebra $\mathcal{T} \mathcal{G} r(G)$. This is the usual Lie algebra defined from the lower central series, truncated at the third rank. For each even Artin group $G$ we determine a presentation for $\mathcal{T} \mathcal{G} r(G)$. Then we prove a criterion to determine whether two Coxeter matrices are isomorphic. Let $c, d \in \mathbb{N}$ such that $c \geq 1, d \geq 2$ and $\operatorname{gcd}(c, d)=1$. We show that, if two even Artin groups $G$ and $G^{\prime}$ having presentations with relations of the form $(s t)^{n}=(t s)^{n}$ with $n \in\{c\} \cup\left\{d^{k} \mid k \geq 1\right\}$ are such that $\mathcal{T} \mathcal{G} r(G) \simeq \mathcal{T} \mathcal{G} r\left(G^{\prime}\right)$, then $G$ and $G^{\prime}$ have the same presentation up to permutation of the generators. On the other hand, we show an example of two non-isomorphic even Artin groups $G$ and $G^{\prime}$ such that $\mathcal{T G} r(G) \simeq \mathcal{T} \mathcal{G} r\left(G^{\prime}\right)$.
\end{abstract}

AMS Subject Classification 20F36

\section{Introduction}

Let $S$ be a finite set. A Coxeter matrix over $S$ is a square matrix $M=\left(m_{s, t}\right)_{s, t \in S}$ indexed by the elements of $S$, with coefficients in $\mathbb{N} \cup\{\infty\}$, satisfying $m_{s, s}=1$ for all $s \in S$, and $m_{s, t}=m_{t, s} \geq 2$ for all $s, t \in S, s \neq t$. For $s, t \in S$ and $m$ an integer greater or equal to 2 we denote by $\Pi(s, t, m)$ the word $s t s \cdots$ of length $m$. In other words, $\Pi(s, t, m)=(s t)^{\frac{m}{2}}$ if $m$ is even and $\Pi(s, t, m)=(s t)^{\frac{m-1}{2}} s$ if $m$ is odd. Let $M=\left(m_{s, t}\right)_{s, t \in S}$ be a Coxeter matrix. The Artin group associated with $M$ is the group $A[M]$ defined by the presentation

$$
\left.A[M]=\langle S| \Pi\left(s, t, m_{s, t}\right)=\Pi\left(t, s, m_{s, t}\right) \text { for } s, t \in S, s \neq t, m_{s, t} \neq \infty\right\rangle .
$$

The Coxeter group $W[M]$ associated with $M$ is the quotient of $A[M]$ by the relations $s^{2}=1, s \in S$. We say that $A[M]$ (or $M$ ) is of spherical type if $W[M]$ is finite, and we say that $A[M]$ (or $M$ ) is right-angled if $m_{s, t} \in\{2, \infty\}$ for all $s, t \in S, s \neq t$. On the other hand, we say that $A[M]$ (or $M$ ) is even if $m_{s, t}$ is even for all $s, t \in S, s \neq t$.

There are few results proven for all Artin groups and the theory consists essentially on the study of more or less extended families. Basic questions such as the existence of torsion or the existence of a solution to the word problem are still open for all these groups. The two most studied and best understood families of Artin groups are the one of Artin groups of spherical type and the one of right-angled Artin groups. The family of even Artin groups has not been so much studied (see, however, Blasco-GarcíaCogolludo-Agustín [3], Blasco-García-Juhász-Paris [4] and Blasco-García-Martínez-Pérez-Paris [5]), but we are convinced by its interest, in particular because even Artin groups share several interesting properties with right-angled Artin groups. For instance, any even Artin group retracts into any of its parabolic subgroups. 
Let $\mathcal{M}$ be a family of Coxeter matrices, and let $\mathcal{A}[\mathcal{M}]=\{A[M] \mid M \in \mathcal{M}\}$ be the corresponding family of Artin groups. A solution to the isomorphism problem in $\mathcal{A}[\mathcal{M}]$ is an algorithm which, given two Coxeter matrices $M, N \in \mathcal{M}$, determines whether $A[M]$ and $A[N]$ are isomorphic or not. On the other hand, we say that the family $\mathcal{A}[\mathcal{M}]$ is rigid if, given two Coxeter matrices $M, N \in \mathcal{M}$, we have $A[M] \simeq A[N]$ if and only if $M \simeq N$. Note that any rigid family of Artin groups has an obvious solution to the isomorphism problem. Note also that these two definitions can be extended to Coxeter groups and, more generally, to any object defined from a Coxeter matrix.

We know that the family of right-angled Artin groups is rigid (see Droms [10]), and we know that the family of Artin groups of spherical type is rigid (see Paris [15]). On the other hand, examples of non-isomorphic Coxeter matrices having isomorphic associated Artin groups can be found in BradyMcCammond-Mühlherr-Neumann [6]. Apart from these works, there are very few significant results on the isomorphism problem for Artin groups.

We know by Bahls [1, Theorem 5.4] that the family of even Coxeter groups is rigid. Furthermore, we do not know any example of two Coxeter matrices $M$ and $N$ such that $A[M]$ is isomorphic to $A[N]$ but $W[M]$ is not isomorphic to $W[N]$. So, for this reason we conjecture that the family of even Artin groups is rigid. This is the question that motivated our study. Note that Bahls' [1] ideas cannot be extended to the study of even Artin groups because they strongly use the study of finite subgroups and we know by Charney [7] and Charney-Davis [8] that even Artin groups have finite cohomological dimension, hence they are torsion free. Our approach here is inspired by the work of Droms [10] and Kim-Makar-Limanov-Neggers [11] on right-angled Artin groups and graph algebras.

Let $G$ be a discrete group and let $\left\{\gamma_{k} G\right\}_{k=1}^{\infty}$ be its lower central series. One can associate to $G$ a graded Lie $\mathbb{Z}$-algebra $\mathcal{G} r(G)=\oplus_{k=1}^{\infty} \mathcal{G} r_{k}(G)$, where $\mathcal{G} r_{k}(G)=\frac{\gamma_{k} G}{\gamma_{k+1} G}$ for all $k$ (see Lazard [13], Magnus-Karrass-Solitar [14], or Bass-Lubotzky [2]). In the present work we use the Lie algebra $\mathcal{G} r(G)$ truncated at the third rank, that is, $\mathcal{T} \mathcal{G} r(G)=\mathcal{G} r(G) / I$, where $I=\oplus_{k=3}^{\infty} \mathcal{G} r_{k}(G)$. Using a variant of Droms' [10] proof based on Kim-Makar-Limanov-Neggers [11] of the rigidity of the family of right-angled Artin groups (see also Koberda [12, Theorem 6.4]), we show that, if $M$ and $N$ are two right-angled Coxeter matrices and $\mathcal{T} \mathcal{G} r(A[M]) \simeq \mathcal{T} \mathcal{G} r(A[N])$, then $M \simeq N$ (see Corollary 4.2). This obviously implies that, if $A[M]$ is isomorphic to $A[N]$, then $M \simeq N$. So, this shows (again) that the family of right-angled Artin groups is rigid. This is the point of view we intend to extend.

In this paper we show that the Lie algebras $\mathcal{T} \mathcal{G} r(G)$ differentiate some even Artin groups but not all of them. In Section 2 we determine a presentation for $\mathcal{T} \mathcal{G} r(A[M])$, where $M$ is any even Coxeter matrix (see Theorem 2.1). For $c, d \in \mathbb{N}$ such that $c \geq 1, d \geq 2$ and $\operatorname{gcd}(c, d)=1$, we denote by $\mathcal{E}(c, d)$ the set of even Coxeter matrices $M=\left(m_{s, t}\right)_{s, t \in S}$ satisfying $m_{s, t} \in\{2 c, \infty\} \cup\left\{2 d^{r} \mid r \geq 1\right\}$ for all $s, t \in S$, $s \neq t$. In Section 5 we prove that the family $\mathcal{A}[\mathcal{E}(c, d)]$ is rigid (see Corollary 5.13), and in Section 6 we show two even Coxeter matrices $M_{0}$ and $N_{0}$ such that $A\left[M_{0}\right] \not \mathcal{A}\left[N_{0}\right]$ and $\mathcal{T} \mathcal{G} r\left(A\left[M_{0}\right]\right) \simeq \mathcal{T} \mathcal{G} r\left(A\left[N_{0}\right]\right)$. The proof of Corollary 5.13 uses a criterion proved in Section 3 to determine whether two Coxeter matrices are isomorphic, as well as a study made in Section 4 of some graded Lie algebras associated to right-angled Coxeter matrices. These two sections may interest some readers regardless of the rest of the paper.

Acknowledgments The authors would like to thank Conchita Martínez Pérez for her comments about the lower central series in even Artin groups which were the seed of this work. The first author 
was partially supported by the Departamento de Industria e Innovación del Gobierno de Aragón and Fondo Social Europeo PhD grant, the Spanish Government MTM2015-67781-P (MINECO/FEDER) and MTM2016-76868-C2-2-P and Grupo Algebra y Geometría from Gobierno de Aragón. He was also supported by the grant CB 2/18 from Programa CAI-Ibercaja de Estancias de Investigación sponsored by Universidad de Zaragoza, Fundación Bancaria Ibercaja and Fundación CAI.

\section{Truncated Lie algebra associated to the lower central series of an even Artin group}

Let $G$ be a group. For $\alpha, \beta \in G$ we denote by $[\alpha, \beta]=\alpha^{-1} \beta^{-1} \alpha \beta$ the commutator of $\alpha$ and $\beta$. If $H_{1}$ and $H_{2}$ are two subgroups of $G$, then we denote by $\left[H_{1}, H_{2}\right]$ the subgroup of $G$ generated by $\left\{[\alpha, \beta] \mid \alpha \in H_{1}, \beta \in H_{2}\right\}$. The lower central series of $G$ is the sequence $\left\{\gamma_{k} G\right\}_{k=1}^{\infty}$ of subgroups of $G$ recursively defined by $\gamma_{1} G=G$ and $\gamma_{k} G=\left[G, \gamma_{k-1} G\right]$ for $k \geq 2$. For $k \geq 1$ the quotient $\mathcal{G} r_{k}(G)=\frac{\gamma_{k} G}{\gamma_{k+1} G}$ is an abelian group, so it is a $\mathbb{Z}$-module. We set $\mathcal{G} r(G)=\oplus_{k=1}^{\infty} \mathcal{G} r_{k}(G)$. For $\alpha \in \gamma_{k} G$, we denote by $\bar{\alpha}$ the element of $\mathcal{G} r_{k}(G)$ represented by $\alpha$. Then $\mathcal{G r}(G)$ is endowed with a structure of graded Lie $\mathbb{Z}$-algebra as follows. If $\alpha \in \gamma_{k} G$ and $\beta \in \gamma_{\ell} G$, then the bracket $[\bar{\alpha}, \bar{\beta}]$ is the element of $\mathcal{G} r_{k+\ell}(G)$ represented by the commutator $[\alpha, \beta]$. We refer to Lazard [13], Magnus-Karrass-Solitar [14], and Bass-Lubotzky [2] for detailed accounts on this Lie algebra.

We denote by $\mathcal{T} \mathcal{G} r(G)=\mathcal{G} r(G) / I$ the quotient of $\mathcal{G} r(G)$ by the ideal $I=\oplus_{k=3}^{\infty} \mathcal{G} r_{k}(G)$. We have $\mathcal{T} \mathcal{G} r(G)=_{\mathbb{Z}} \mathcal{G} r_{1}(G) \oplus \mathcal{G} r_{2}(G)$, and the Lie bracket on $\mathcal{T} \mathcal{G} r(G)$ is defined as follows. If $\alpha \in \gamma_{k} G$ and $\beta \in \gamma_{\ell} G, 1 \leq k, \ell \leq 2$, then $[\bar{\alpha}, \bar{\beta}]$ is the element of $\mathcal{G} r_{k+\ell}(G)$ represented by $[\alpha, \beta]$ if $k=\ell=1$, and $[\bar{\alpha}, \bar{\beta}]=0$ otherwise. It is clear that, if $G$ is isomorphic to $G^{\prime}$, then $\mathcal{T} \mathcal{G} r(G)$ is isomorphic to $\mathcal{T} \mathcal{G} r\left(G^{\prime}\right)$.

Now, take an even Coxeter matrix $M=\left(m_{s, t}\right)_{s, t \in S}$ and fix a total order $<$ on $S$. For $m \in \mathbb{N} \cup\{\infty\}$ we set $E_{m}(M)=\left\{(s, t) \in S \times S \mid s<t\right.$ and $\left.m_{s, t}=m\right\}$. We set

$$
\begin{gathered}
L_{2}[M]=\left(\bigoplus_{2 \leq n<\infty}\left(\bigoplus_{(s, t) \in E_{2 n}(M)}(\mathbb{Z} / n \mathbb{Z}) v_{s, t}\right)\right) \oplus\left(\bigoplus_{(s, t) \in E_{\infty}(M)} \mathbb{Z} v_{s, t}\right), \\
L_{1}[M]=\bigoplus_{s \in S} \mathbb{Z} u_{s}, L[M]=L_{1}[M] \oplus L_{2}[M] .
\end{gathered}
$$

We define a Lie bracket $[\cdot, \cdot]$ on $L[M]$ as follows. Let $s, t \in S, s \neq t$. Then $\left[u_{s}, u_{t}\right]=-\left[u_{t}, u_{s}\right]=v_{s, t}$ if $s<t$ and $m_{s, t} \neq 2$, and $\left[u_{s}, u_{t}\right]=0$ if $m_{s, t}=2$. On the other hand, we set $[\alpha, \beta]=0$ for each $(\alpha, \beta) \in L[M] \times L_{2}[M]$. It is clear that $L[M]$ is a well-defined graded Lie $\mathbb{Z}$-algebra.

Theorem 2.1 Let $M$ be an even Coxeter matrix. Then $\mathcal{T} \mathcal{G} r(A[M])$ is isomorphic to $L[M]$.

Proof Claim 1. Let $G$ be a group, let $\alpha, \beta \in G$, and let $n \in \mathbb{N}, n \geq 1$. Then

$$
(\beta \alpha)^{-n}(\alpha \beta)^{n} \equiv[\alpha, \beta]^{n}\left(\bmod \gamma_{3} G\right) .
$$

Proof of Claim 1. We argue by induction on $n$. If $n=1$, then $(\beta \alpha)^{-1}(\alpha \beta)=[\alpha, \beta]$ and there is nothing to prove. We suppose that $n \geq 2$ and that the inductive hypothesis holds. Then

$$
(\beta \alpha)^{-n}(\alpha \beta)^{n} \equiv \alpha^{-1} \beta^{-1}[\alpha, \beta]^{n-1} \alpha \beta \equiv[\alpha, \beta]^{n-1} \alpha^{-1} \beta^{-1} \alpha \beta \equiv[\alpha, \beta]^{n}\left(\bmod \gamma_{3} G\right) .
$$


This concludes the proof of Claim 1.

Claim 2. There is a surjective homomorphism $f: L[M] \rightarrow \mathcal{T} \mathcal{G} r(A[M])$ which sends $u_{s}$ to $\bar{s}$ for all $s \in S$ and sends $v_{s, t}$ to $[\bar{s}, \bar{t}]$ for all $s, t \in S$ such that $s<t$ and $m_{s, t} \neq 2$.

Proof of Claim 2. We know by Magnus-Karrass-Solitar [14, Theorem 5.4] that $\mathcal{T} \mathcal{G} r_{1}(A[M])$ is generated as a $\mathbb{Z}$-module by $\{\bar{s} \mid s \in S\}$ and $\mathcal{T} \mathcal{G}_{2}(A[M])$ is generated as a $\mathbb{Z}$-module by $\{[\bar{s}, \bar{t}] \mid s, t \in$ $S, s<t\}$. Let $s, t \in S, s<t$. If $m_{s, t}=2$, then $s t=t s$, hence $[\bar{s}, \bar{t}]=0$. If $m_{s, t}=2 n$, where $2 \leq n<\infty$, then $(s t)^{n}=\Pi\left(s, t, m_{s, t}\right)=\Pi\left(t, s, m_{s, t}\right)=(t s)^{n}$, hence, by Claim $1, n[\bar{s}, \bar{t}]=0$. So, there exists a well-defined homomorphism $f: L[M] \rightarrow \mathcal{T} \mathcal{G} r(A[M])$ which sends $u_{s}$ to $\bar{s}$ for all $s \in S$ and sends $v_{s, t}$ to $[\bar{s}, \bar{t}]$ for all $s, t \in S$ such that $s<t$ and $m_{s, t} \neq 2$, and this homomorphism is surjective. This concludes the proof of Claim 2.

Let $\mathbb{Z}\langle X\rangle$ be the non-commutative associative free algebra over a set $X=\left\{x_{s} \mid s \in S\right\}$ in one-to-one correspondence with $S$. Let $\hat{I}$ be the ideal of $\mathbb{Z}\langle X\rangle$ generated by $X$, and let $\hat{R}=\mathbb{Z}\langle X\rangle / \hat{I}^{3}$. Let $J$ be the ideal of $\hat{R}$ generated by $\cup_{1 \leq n<\infty}\left\{n\left(x_{s} x_{t}-x_{t} x_{s}\right) \mid(s, t) \in E_{2 n}(M)\right\}$, and let $R=\hat{R} / J$. Observe that $R$ is a graded associative $\mathbb{Z}$-algebra, $R=R_{0} \oplus R_{1} \oplus R_{2}$, where $R_{0}=\mathbb{Z}, R_{1}=\oplus_{s \in S} \mathbb{Z} x_{s}$, and

$$
\begin{gathered}
R_{2}=\left(\bigoplus_{s \in S} \mathbb{Z} x_{s}^{2}\right) \oplus\left(\bigoplus_{s<t} \mathbb{Z} x_{t} x_{s}\right) \oplus\left(\bigoplus_{2 \leq n<\infty}\left(\bigoplus_{(s, t) \in E_{2 n}(M)}(\mathbb{Z} / n \mathbb{Z})\left(x_{s} x_{t}-x_{t} x_{s}\right)\right)\right) \\
\oplus\left(\bigoplus_{(s, t) \in E_{\infty}(M)} \mathbb{Z}\left(x_{s} x_{t}-x_{t} x_{s}\right)\right) .
\end{gathered}
$$

Let $I=R_{1} \oplus R_{2}$ be the ideal of $R$ generated by $X$. Set $\mathcal{U}(R)=\{1+w \mid w \in I\}$. Note that $\mathcal{U}(R)$ is a subgroup of the group of units of $R$; the inverse of an element $1+w$ of $\mathcal{U}(R)$ is $1-w+w^{2}$.

Claim 3. Let $u, v \in I$ and $n \in \mathbb{N}, n \geq 1$. Then

$$
((1+u)(1+v))^{n}=1+n u+n v+S_{n-1}\left(u^{2}+v^{2}+v u\right)+S_{n} u v,
$$

where $S_{n}=\sum_{k=1}^{n} k=\frac{n(n+1)}{2}$.

Proof of Claim 3. We argue by induction on $n$. The case $n=1$ being trivial, we may assume that $n \geq 2$ and that the inductive hypothesis holds. Then

$$
\begin{gathered}
((1+u)(1+v))^{n} \\
=\left(1+(n-1) u+(n-1) v+S_{n-2}\left(u^{2}+v^{2}+v u\right)+S_{n-1} u v\right)(1+u+v+u v) \\
=1+(n-1) u+(n-1) v+S_{n-2}\left(u^{2}+v^{2}+v u\right)+S_{n-1} u v+u+(n-1) u^{2}+(n-1) v u \\
+v+(n-1) u v+(n-1) v^{2}+u v \\
=1+n u+n v+S_{n-1}\left(u^{2}+v^{2}+v u\right)+S_{n} u v .
\end{gathered}
$$

This concludes the proof of Claim 3.

Claim 4. There exists a homomorphism $\tilde{g}: A[M] \rightarrow \mathcal{U}(R)$ which sends $s$ to $1+x_{s}$ for all $s \in S$. 
Proof of Claim 4. Let $s, t \in S, s \neq t$, such that $m_{s, t} \neq \infty$. Set $m_{s, t}=2 n$ where $1 \leq n<\infty$. Then, by Claim 3,

$$
\begin{gathered}
\left(\left(1+x_{s}\right)\left(1+x_{t}\right)\right)^{n}-\left(\left(1+x_{t}\right)\left(1+x_{s}\right)\right)^{n} \\
=\left(1+n x_{s}+n x_{t}+S_{n-1}\left(x_{s}^{2}+x_{t}^{2}+x_{t} x_{s}\right)+S_{n} x_{s} x_{t}\right) \\
-\left(1+n x_{t}+n x_{s}+S_{n-1}\left(x_{t}^{2}+x_{s}^{2}+x_{s} x_{t}\right)+S_{n} x_{t} x_{s}\right) \\
=n\left(x_{s} x_{t}-x_{t} x_{s}\right)=0,
\end{gathered}
$$

hence

$$
\begin{aligned}
& \Pi\left(\left(1+x_{s}\right),\left(1+x_{t}\right), m_{s, t}\right)=\left(\left(1+x_{s}\right)\left(1+x_{t}\right)\right)^{n} \\
= & \left(\left(1+x_{t}\right)\left(1+x_{s}\right)\right)^{n}=\Pi\left(\left(1+x_{t}\right),\left(1+x_{s}\right), m_{s, t}\right) .
\end{aligned}
$$

This concludes the proof of Claim 4.

We denote by $g: \mathcal{T G} r(A[M]) \rightarrow \mathcal{T G} r(\mathcal{U}(R))$ the homomorphism induced by $\tilde{g}$. For $k \geq 1$ we set $\mathcal{U}_{k}(R)=\left\{1+w \mid w \in I^{k}\right\}$. Note that $\mathcal{U}_{k}(R)$ is a subgroup of $\mathcal{U}(R)$ and $\mathcal{U}_{k}(R)=\{1\}$ if $k \geq 3$.

Claim 5.

(1) Let $k \geq 1$. Then $\mathcal{U}_{k}(R)$ is a normal subgroup of $\mathcal{U}(R)$.

(2) Let $k, \ell \geq 1$. Then $\left[\mathcal{U}_{k}(R), \mathcal{U}_{\ell}(R)\right] \subset \mathcal{U}_{k+\ell}(R)$.

Proof of Claim 5. Let $\alpha \in \mathcal{U}(R)$ and $w \in I^{k}$. Then $\alpha(1+w) \alpha^{-1}=1+\alpha w \alpha^{-1} \in \mathcal{U}_{k}(R)$. So, $\mathcal{U}_{k}(R)$ is a normal subgroup of $\mathcal{U}(R)$.

Let $k, \ell \geq 1, u \in I^{k}$ and $v \in I^{\ell}$. Then

$$
\begin{gathered}
{[(1+u),(1+v)]=(1+u)^{-1}(1+v)^{-1}(1+u)(1+v)} \\
=\left(1-u-v+u^{2}+v^{2}+u v\right)(1+u+v+u v) \\
=1-u-v+u^{2}+v^{2}+u v+u-u^{2}-v u+v-u v-v^{2}+u v \\
=1+u v-v u \in \mathcal{U}_{k+\ell}(R) .
\end{gathered}
$$

This concludes the proof of Claim 5.

End of the proof of Theorem 2.1. By Claim 5 we have $\gamma_{k} \mathcal{U}(R) \subset \mathcal{U}_{k}(R)$ for all $k \in \mathbb{N}$, and these inclusions induce for each $k$ a homomorphism $\mathcal{G} r_{k}(\mathcal{U}(R))=\frac{\gamma_{k} \mathcal{U}(R)}{\gamma_{k+1} \mathcal{U}(R)} \rightarrow \frac{\mathcal{U}_{k}(R)}{\mathcal{U}_{k+1}(R)}$ of $\mathbb{Z}$-modules. On the other hand, it is easily seen that $\frac{\mathcal{U}_{k}(R)}{\mathcal{U}_{k+1}(R)}$ is isomorphic to $R_{k}$ as a $\mathbb{Z}$-module, where $R_{k}=\{0\}$ if $k \geq 3$. For $k=1,2$, by composing these homomorphisms we obtain a homomorphism $h_{k}: \mathcal{T} \mathcal{G} r_{k}(\mathcal{U}(R))=$ $\mathcal{G} r_{k}(\mathcal{U}(R)) \rightarrow R_{k}$ of $\mathbb{Z}$-modules. A direct calculation shows that $\left(h_{1} \circ g \circ f\right)\left(u_{s}\right)=x_{s}$ for all $s \in S$, hence $h_{1} \circ g \circ f: L_{1}[M] \rightarrow R_{1}$ is injective, and therefore the restriction of $f$ to $L_{1}[M]$ is injective. Similarly, a direct calculation shows that, for each $s, t \in S$ such that $s<t$ and $m_{s, t} \neq 2$, we have $\left(h_{2} \circ g \circ f\right)\left(v_{s, t}\right)=\left(x_{s} x_{t}-x_{t} x_{s}\right)$, hence $h_{2} \circ g \circ f: L_{2}[M] \rightarrow R_{2}$ is injective, and therefore the restriction of $f$ to $L_{2}[M]$ is injective. So, $f$ is an isomorphism. 


\section{Isomorphism between Coxeter matrices}

Let $M$ and $N$ be two Coxeter matrices. In the present section we prove a necessary and sufficient condition for $M$ and $N$ to be isomorphic (see Proposition 3.4). This condition will be used later for even Coxeter matrices but it holds for any pair of Coxeter matrices. The case of right-angled Coxeter matrices is well-known and proved in Kim-Makar-Limanov-Neggers-Roush [11], with a proof that inspired ours.

Let $M=\left(m_{s, t}\right)_{s, t \in S}$ be a Coxeter matrix. For $s \in S$ and $m \in \mathbb{N}, 2 \leq m<\infty$, the set $\operatorname{lk}_{m}(s)=\{t \in$ $S \backslash\{s\} \mid m_{s, t}$ divides $\left.m\right\}$ is called the $m$-link of $s$ and the set $\operatorname{st}_{m}(s)=\operatorname{lk}_{m}(s) \cup\{s\}$ is called the $m$-star of $s$. For $s, t \in S$ we set $s \prec_{M} t$ if $\mathrm{lk}_{m}(s) \subset \mathrm{st}_{m}(t)$ for all $m \in \mathbb{N}, 2 \leq m<\infty$.

Lemma 3.1 The relation $\prec_{M}$ is a quasi-order.

Proof The relation $\prec_{M}$ is clearly reflexive. So, we just need to show that $\prec_{M}$ is transitive. Let $s, t, r \in S$ such that $s \prec_{M} t$ and $t \prec_{M} r$. Let $m \in \mathbb{N}$ such that $2 \leq m<\infty$. We have $\operatorname{lk}_{m}(s) \subset \operatorname{st}_{m}(t)$ and $\mathrm{lk}_{m}(t) \subset \mathrm{st}_{m}(r)$, and we have to show that $\mathrm{lk}_{m}(s) \subset \mathrm{st}_{m}(r)$. If $s=t$ or $t=r$ or $s=r$, then there is nothing to prove, thus we may assume that $s \neq t, t \neq r$ and $s \neq r$. If $m_{s, t}$ does not divide $m$, then $t \notin \mathrm{lk}_{m}(s)$, hence $\mathrm{lk}_{m}(s) \subset \mathrm{lk}_{m}(t)$, and therefore $\mathrm{lk}_{m}(s) \subset \mathrm{st}_{m}(r)$. So, we can suppose that $m_{s, t}$ divides $m$, that is, $s \in \mathrm{lk}_{m}(t)$ and $t \in \mathrm{lk}_{m}(s)$. Since $\mathrm{lk}_{m}(t) \subset \mathrm{st}_{m}(r)$ and $s \neq r$, it follows that $s \in \mathrm{lk}_{m}(r)$, hence $m_{s, r}$ divides $m$ and $r \in \mathrm{lk}_{m}(s)$. Since $\mathrm{lk}_{m}(s) \subset \mathrm{st}_{m}(t)$ and $r \neq t$, it follows that $r \in \mathrm{lk}_{m}(t)$, hence $m_{t, r}$ divides $m$ and $t \in \mathrm{kk}_{m}(r)$. So, $\mathrm{lk}_{m}(s) \backslash\{t\} \subset \mathrm{lk}_{m}(t) \subset \mathrm{st}_{m}(r)$ and $t \in \mathrm{lk}_{m}(r) \subset \mathrm{st}_{m}(r)$, hence $\mathrm{lk}_{m}(s) \subset \mathrm{st}_{m}(r)$.

Define the equivalence relation $\equiv_{M}$ on $S$ by

$$
s \equiv_{M} t \Longleftrightarrow s \prec_{M} t \text { and } t \prec_{M} s .
$$

Lemma 3.2 Let $C$ be an equivalence class of $\equiv_{M}$ non reduced to a single element. Then there exists $m \in \mathbb{N} \cup\{\infty\}, m \geq 2$, such that $m_{s, t}=m$ for all $s, t \in C, s \neq t$.

Proof Let $s, t \in C, s \neq t$, and let $m=m_{s, t}$. Let $r \in C \backslash\{s, t\}$. Suppose first that $m \neq \infty$. We have $t \in \mathrm{lk}_{m}(s)$ and $\mathrm{lk}_{m}(s) \subset \mathrm{st}_{m}(r)$, hence $t \in \mathrm{st}_{m}(r)$. Since $t \neq r$, it follows that $t \in \mathrm{lk}_{m}(r)$, thus $m_{t, r}$ divides $m=m_{s, t}$. In particular, $m_{t, r} \neq \infty$. Using the same argument we show that $m=m_{s, t}$ divides $m_{t, r}$, hence $m_{t, r}=m_{s, t}=m$. We show that $m_{s, r}=m$ in the same way. Now suppose that $m=\infty$. If we had $m_{s, r} \neq \infty$, then, by the above, we would have $m_{s, t}=m_{s, r} \neq \infty$, a contradiction. Thus $m_{s, r}=\infty$. Similarly, $m_{t, r}=\infty$.

Lemma 3.3 Let $C, D$ be two distinct equivalence classes of $\equiv_{M}$. Then there exists $m \in \mathbb{N} \cup\{\infty\}$, $m \geq 2$, such that $m_{s, t}=m$ for all $s \in C$ and $t \in D$.

Proof We choose $s \in C$ and $t \in D$ and we set $m=m_{s, t}$. We take $t^{\prime} \in D, t^{\prime} \neq t$, and we turn to show that $m_{s, t^{\prime}}=m$. Suppose first that $m \neq \infty$. Since $C$ and $D$ are different, we have $s \neq t$ and $s \neq t^{\prime}$. Moreover, $s \in \mathrm{lk}_{m}(t)$ and $\mathrm{lk}_{m}(t) \subset \mathrm{st}_{m}\left(t^{\prime}\right)$, hence $s \in \mathrm{lk}_{m}\left(t^{\prime}\right)$, and therefore $m_{s, t^{\prime}}$ divides $m=m_{s, t}$. In particular $m_{s, t^{\prime}} \neq \infty$. We show that $m_{s, t}$ divides $m_{s, t^{\prime}}$ with the same argument, hence $m_{s, t^{\prime}}=m_{s, t}=m$. Now, suppose that $m=\infty$. If we had $m_{s, t^{\prime}} \neq \infty$, then, by the above, we would have $m_{s, t}=m_{s, t^{\prime}} \neq \infty$, a contradiction. So, $m_{s, t^{\prime}}=\infty$. 
Thanks to Lemma 3.3 we can define from $M$ a Coxeter matrix $M^{r}=\left(m_{C, D}^{r}\right)_{C, D \in S^{r}}$ as follows.

(a) $S^{r}$ is the set of equivalence classes of $\equiv_{M}$.

(b) Let $C, D \in S^{r}$. Then choose $s \in C$ and $t \in D$, and set $m_{C, D}^{r}=m_{s, t}$.

Furthermore, thanks to Lemma 3.2 we can define a map lb : $S^{r} \rightarrow \mathbb{N} \cup\{\infty\}$ as follows. Let $C \in S^{r}$. If $|C|=1$ we set $\operatorname{lb}(C)=0$. If $|C| \geq 2$ we set $\operatorname{lb}(C)=m_{s, t}$, where $s, t$ are two distinct elements of $C$.

Proposition 3.4 Let $M=\left(m_{s, t}\right)_{s, t \in S}$ and $N=\left(n_{x, y}\right)_{x, y \in T}$ be two Coxeter matrices. If there exists an isomorphism $f: M^{r} \rightarrow N^{r}$ such that $|C|=|f(C)|$ and $\operatorname{lb}(C)=\operatorname{lb}(f(C))$ for all $C \in S^{r}$, then $M$ and $N$ are isomorphic.

Proof For each $C \in S^{r}$, since $|C|=|f(C)|$, we can choose a bijection $\hat{f}_{C}: C \rightarrow f(C)$. Then we define $\hat{f}: S \rightarrow T$ as follows. Let $s \in S$. Let $C \in S^{r}$ such that $s \in C$. Then we set $\hat{f}(s)=\hat{f}_{C}(s)$. The map $\hat{f}: S \rightarrow T$ is a bijection by construction. Moreover, it is easily seen that $m_{s, t}=n_{\hat{f}(s) \hat{f}(t)}$ for all $s, t \in S$, hence $\hat{f}$ is an isomorphism from $M$ to $N$.

\section{Lie algebras associated to right-angled Artin groups}

Let $M=\left(m_{s, t}\right)_{s, t \in S}$ be a right-angled Coxeter matrix. We assume $S$ to be endowed with a total order $\leq$. Let $\mathbb{K}$ be a field. Recall that, for $m \in\{2, \infty\}$, we set $E_{m}(M)=\left\{(s, t) \in S \times S \mid s<t\right.$ and $\left.m_{s, t}=m\right\}$. We set

$$
L_{\mathbb{K}, 1}[M]=\bigoplus_{s \in S} \mathbb{K} u_{s}, L_{\mathbb{K}, 2}[M]=\bigoplus_{(s, t) \in E_{\infty}(M)} \mathbb{K} v_{s, t}, L_{\mathbb{K}}[M]=L_{\mathbb{K}, 1}[M] \oplus L_{\mathbb{K}, 2}[M] .
$$

We define a Lie bracket $[\cdot, \cdot]$ on $L_{\mathbb{K}}[M]$ as follows. Let $s, t \in S, s \neq t$. Then $\left[u_{s}, u_{t}\right]=-\left[u_{t}, u_{s}\right]=v_{s, t}$ if $s<t$ and $m_{s, t}=\infty$, and $\left[u_{s}, u_{t}\right]=0$ if $m_{s, t}=2$. On the other hand, we set $[\alpha, \beta]=0$ for all $(\alpha, \beta) \in L_{\mathbb{K}}[M] \times L_{\mathbb{K}, 2}[M]$. It is clear that $L_{\mathbb{K}}[M]$ is a well-defined graded Lie $\mathbb{K}$-algebra. In fact, $L_{\mathbb{K}}[M]=\mathbb{K} \otimes L[M]$. The goal of the present section is to prove the following theorem.

Theorem 4.1 Let $M$ and $N$ be two right-angled Coxeter matrices and let $\mathbb{K}$ be a field. If $L_{\mathbb{K}}[M]$ and $L_{\mathbb{K}}[N]$ are isomorphic, then $M$ and $N$ are isomorphic.

Corollary 4.2 Let $M$ and $N$ be two right-angled Coxeter matrices. If $L[M]$ and $L[N]$ are isomorphic, then $M$ and $N$ are isomorphic.

Proof Let $M$ and $N$ be two right-angled Coxeter matrices such that $L[M]$ and $L[N]$ are isomorphic. Then $\mathbb{Q} \otimes L[M]=L_{\mathbb{Q}}[M]$ is isomorphic to $\mathbb{Q} \otimes L[N]=L_{\mathbb{Q}}[N]$, hence, by Theorem 4.1, $M$ and $N$ are isomorphic.

Corollary 4.3 (Droms [10]) Let $\mathcal{E}_{r a}$ be the set of right-angled Coxeter matrices. Then $\mathcal{A}\left[\mathcal{E}_{r a}\right]$ is rigid. 
Proof Let $M$ and $N$ be two right-angled Coxeter matrices such that $A[M]$ and $A[N]$ are isomorphic. Then $\mathcal{T} \mathcal{G} r(A[M])$ and $\mathcal{T} \mathcal{G} r(A[N])$ are isomorphic, hence, by Theorem 2.1, $L[M]$ and $L[N]$ are isomorphic, and therefore, by Corollary 4.2, $M$ and $N$ are isomorphic.

The remaining of the section is devoted to the proof of Theorem 4.1.

Let $M=\left(m_{s, t}\right)_{s, t \in S}$ be a right-angled Coxeter matrix. We associate to $M$ a graph $\Gamma_{\infty}[M]$ as follows.

(a) $S$ is the set of vertices of $\Gamma_{\infty}[M]$.

(b) Two vertices $s, t \in S, s \neq t$, are connected by an edge if $m_{s, t}=\infty$.

For $X \subset S$ we denote by $\Gamma_{\infty, X}[M]$ the full subgraph of $\Gamma_{\infty}[M]$ spanned by $X$. On the other hand, for $X \subset S$ we set $\operatorname{lk}_{2}(X)=\cap_{s \in X} \operatorname{lk}_{2}(s)$.

Let $\mathbb{K}$ be a field. The support of an element $\alpha=\sum_{s \in S} a_{s} u_{s} \in L_{\mathbb{K}, 1}[M]$ is defined to be $\operatorname{supp}(\alpha)=$ $\left\{s \in S \mid a_{s} \neq 0\right\}$. On the other hand, the centralizer of an element $\alpha \in L_{\mathbb{K}, 1}[M]$ is $Z(\alpha)=\{\beta \in$ $\left.L_{\mathbb{K}, 1}[M] \mid[\alpha, \beta]=0\right\}$.

Lemma 4.4 Let $\mathbb{K}$ be a field, let $M=\left(m_{s, t}\right)_{s, t \in S}$ be a right-angled Coxeter matrix, and let $\alpha=$ $\sum_{s \in S} a_{s} u_{s} \in L_{\mathbb{K}, 1}[M]$. Let $X=\operatorname{supp}(\alpha)$, and let $\Omega_{1}, \ldots, \Omega_{\ell}$ be the connected components of $\Gamma_{\infty, X}[M]$. For each $i \in\{1, \ldots, \ell\}$ we denote by $X_{i}$ the set of vertices of $\Omega_{i}$ and we set $\alpha_{i}=\sum_{s \in X_{i}} a_{s} u_{s}$. Then

$$
Z(\alpha)=\left(\bigoplus_{i=1}^{\ell} \mathbb{K} \alpha_{i}\right) \oplus\left(\bigoplus_{s \in \mathrm{k}_{2}(X)} \mathbb{K} u_{s}\right) .
$$

Proof Set

$$
V=\left(\bigoplus_{i=1}^{\ell} \mathbb{K} \alpha_{i}\right) \oplus\left(\bigoplus_{s \in \mathrm{k}_{2}(X)} \mathbb{K} u_{s}\right) .
$$

The inclusion $V \subset Z(\alpha)$ is easily checked, so we just need to show that $Z(\alpha) \subset V$. Let $\beta=$ $\sum_{s \in S} b_{s} u_{s} \in Z(\alpha)$. We have

$$
0=[\alpha, \beta]=\sum_{(s, t) \in E_{\infty}(M)}\left(a_{s} b_{t}-a_{t} b_{s}\right) v_{s, t} .
$$

Let $s \in S$ such that $s \notin X$ and $s \notin \mathrm{lk}_{2}(X)$. There exists $t \in X$ such that $m_{s, t}=\infty$. Suppose that $s<t$, that is, $(s, t) \in E_{\infty}(M)$. By Equation (4-1), $a_{t} b_{s}=0$ since $a_{s}=0$, hence $b_{s}=0$ since $a_{t} \neq 0$. Similarly, if $t<s$, then $b_{s}=0$. Let $i \in\{1, \ldots, \ell\}$. If $s, t \in X_{i}$ are such that $(s, t) \in E_{\infty}(M)$, then, by Equation (4-1), $a_{s} b_{t}=a_{t} b_{s}$, hence $\frac{b_{s}}{a_{s}}=\frac{b_{t}}{a_{t}}$. Since $\Omega_{i}$ is connected, it follows that $\frac{b_{s}}{a_{s}}=\frac{b_{t}}{a_{t}}$ for all $s, t \in X_{i}$. We choose $s \in X_{i}$ and we set $c_{i}=\frac{b_{s}}{a_{s}}$. Then $\sum_{t \in X_{i}} b_{t} u_{t}=c_{i} \alpha_{i}$. So, $\beta=\left(\sum_{i=1}^{\ell} c_{i} \alpha_{i}\right)+\left(\sum_{s \in \mathrm{k}_{2}(X)} b_{s} u_{s}\right) \in V$.

From now on we assume given a field $\mathbb{K}$, two right-angled Coxeter matrices $M=\left(m_{s, t}\right)_{s, t \in S}$ and $N=\left(n_{x, y}\right)_{x, y \in T}$, and an isomorphism $f: L_{\mathbb{K}}[M] \rightarrow L_{\mathbb{K}}[N]$. Our aim is to prove that $M$ and $N$ are isomorphic. 
Lemma 4.5 Let $s \in S$ and $x, y \in T$ such that $n_{x, y}=2$ and $s \in \operatorname{supp}\left(f^{-1}\left(u_{x}\right)\right)$. Then $\left[u_{y}, f\left(u_{s}\right)\right]=0$.

Proof We set $f^{-1}\left(u_{x}\right)=\sum_{t \in S} a_{t} u_{t}$. Let $X=\operatorname{supp}\left(f^{-1}\left(u_{x}\right)\right)$, and let $\Omega_{1}, \ldots, \Omega_{\ell}$ be the connected components of $\Gamma_{\infty, X}[M]$. For each $i \in\{1, \ldots, \ell\}$ we denote by $X_{i}$ the set of vertices of $\Omega_{i}$, and we set $\alpha_{i}=\sum_{t \in X_{i}} a_{t} u_{t}$. We have $\left[u_{x}, u_{y}\right]=0$, hence $\left[f^{-1}\left(u_{x}\right), f^{-1}\left(u_{y}\right)\right]=0$, and therefore, by Lemma $4.4, f^{-1}\left(u_{y}\right)$ is written in the form $f^{-1}\left(u_{y}\right)=\sum_{i=1}^{\ell} c_{i} \alpha_{i}+\gamma$, where $c_{1}, \ldots, c_{\ell} \in \mathbb{K}$ and $\operatorname{supp}(\gamma) \subset \mathrm{lk}_{2}(X)$. Since $s \in \operatorname{supp}\left(f^{-1}\left(u_{x}\right)\right)=X$, there exits $j \in\{1, \ldots, \ell\}$ such that $s \in X_{j}$. Then $\left[f^{-1}\left(u_{y}\right)-c_{j} f^{-1}\left(u_{x}\right), u_{s}\right]=0$, hence $\left[u_{y}-c_{j} u_{x}, f\left(u_{s}\right)\right]=0$. If $c_{j}=0$, then the above equality becomes $\left[u_{y}, f\left(u_{s}\right)\right]=0$ and there is nothing else to prove. So, we may assume that $c_{j} \neq 0$. Since $n_{x, y}=2$, by applying again Lemma 4.4 we get $f\left(u_{s}\right)=b_{1} u_{y}+b_{2} c_{j} u_{x}+\gamma^{\prime}$, where $b_{1}, b_{2} \in \mathbb{K}$ and $\operatorname{supp}\left(\gamma^{\prime}\right) \subset \mathrm{lk}_{2}(\{x, y\})$. Then $\operatorname{supp}\left(f\left(u_{s}\right)\right) \subset \operatorname{st}_{2}\left(u_{y}\right)$, hence $\left[u_{y}, f\left(u_{s}\right)\right]=0$ in this case as well.

For $s \in S$ and $x \in T$ we set $s \leftrightarrow_{f} x$ if $s \in \operatorname{supp}\left(f^{-1}\left(u_{x}\right)\right)$ and $x \in \operatorname{supp}\left(f\left(u_{s}\right)\right)$.

Lemma 4.6 Let $s \in S$. Then there exists $x \in T$ such that $s \leftrightarrow_{f} x$.

Proof For $s \in S$ we set $f\left(u_{s}\right)=\sum_{x \in T} a_{s, x} u_{x}$ and for $x \in T$ we set $f^{-1}\left(u_{x}\right)=\sum_{s \in S} b_{x, s} u_{s}$. Let $s \in S$. Then

$$
u_{s}=\left(f^{-1} \circ f\right)\left(u_{s}\right)=\sum_{x \in T} \sum_{t \in S} a_{s, x} b_{x, t} u_{t},
$$

hence there exists $x \in T$ such that $a_{s, x} \neq 0$ and $b_{x, s} \neq 0$. Then $s \in \operatorname{supp}\left(f^{-1}\left(u_{x}\right)\right)$ and $x \in \operatorname{supp}\left(f\left(u_{s}\right)\right)$, that is, $s \leftrightarrow_{f} x$.

Lemma 4.7 Let $s, t \in S, s \neq t$, and $x, y \in T, x \neq y$, such that $s \leftrightarrow_{f} x$ and $t \leftrightarrow_{f} y$. We have $m_{s, t}=2$ if and only if $n_{x, y}=2$.

Proof Suppose that $n_{x, y}=2$. Since $s \leftrightarrow_{f} x$, we have $s \in \operatorname{supp}\left(f^{-1}\left(u_{x}\right)\right)$. Then, by Lemma 4.5, $\left[u_{y}, f\left(u_{s}\right)\right]=0$, hence $\left[f^{-1}\left(u_{y}\right), u_{s}\right]=0$. By Lemma 4.4 this implies that $\operatorname{supp}\left(f^{-1}\left(u_{y}\right)\right) \subset \operatorname{st}_{2}(s)$. Since $t \in \operatorname{supp}\left(f^{-1}\left(u_{y}\right)\right)$ and $t \neq s$ it follows that $t \in \mathrm{lk}_{2}(s)$, that is, $m_{s, t}=2$. We show in the same way that, if $m_{s, t}=2$, then $n_{x, y}=2$.

Lemma 4.8 Let $s, t \in S$ and $x, y \in T$ such that $s \leftrightarrow_{f} x$ and $t \leftrightarrow_{f} y$. We have $s \prec_{M} t$ if and only if $x \prec_{N} y$.

Proof We suppose that $s \prec_{M} t$ and turn to prove that $x \prec_{N} y$. If $x=y$, then there is nothing to prove. So, we can assume that $x \neq y$. Let $z \in \mathrm{lk}_{2}(x)$. We have $n_{x, z}=2$ and $s \in \operatorname{supp}\left(f^{-1}\left(u_{x}\right)\right)$ hence, by Lemma 4.5, $\left[u_{z}, f\left(u_{s}\right)\right]=0$, hence $\left[f^{-1}\left(u_{z}\right), u_{s}\right]=0$. By Lemma 4.4 it follows that $f^{-1}\left(u_{z}\right)$ is of the form $f^{-1}\left(u_{z}\right)=a u_{s}+\gamma$ where $a \in \mathbb{K}$ and $\operatorname{supp}(\gamma) \subset \mathrm{lk}_{2}(s)$. Since $s \prec_{M} t$, we have $\mathrm{lk}_{2}(s) \subset \mathrm{st}_{2}(t)$, hence $\left[\gamma, u_{t}\right]=0$, and therefore $\left[u_{z}, f\left(u_{t}\right)\right]=\left[f\left(a u_{s}+\gamma\right), f\left(u_{t}\right)\right]=f\left(\left[a u_{s}+\gamma, u_{t}\right]\right)=a f\left(\left[u_{s}, u_{t}\right]\right)$.

Suppose that $s \neq t, m_{s, t}=\infty$ and $a \neq 0$. We have $t \notin \operatorname{supp}(\gamma) \operatorname{since} \operatorname{supp}(\gamma) \subset \mathrm{k}_{2}(s)$. Let $r \in \operatorname{supp}(\gamma)$. We have $r \in \mathrm{lk}_{2}(s) \subset \mathrm{st}_{2}(t)$ and $r \neq t$, hence $n_{r, t}=2$. Moreover, $y \in \operatorname{supp}\left(f\left(u_{t}\right)\right)$, hence, by Lemma 4.5, $\left[u_{r}, f^{-1}\left(u_{y}\right)\right]=0$. So, $\left[\gamma, f^{-1}\left(u_{y}\right)\right]=0$, hence $\left[f(\gamma), u_{y}\right]=0$. Since $x \in \operatorname{supp}\left(f\left(u_{s}\right)\right)$ 
and $z \neq x$, the equality $u_{z}=a f\left(u_{s}\right)+f(\gamma)$ implies that $x \in \operatorname{supp}(f(\gamma))$. By Lemma 4.4 it follows that $x \in \mathrm{lk}_{2}(y)$, that is, $n_{x, y}=2$. By Lemma 4.7 we deduce that $m_{s, t}=2$, a contradiction.

So, either $s=t$, or $m_{s, t}=2$, or $a=0$. In all theses cases we have $\left[u_{z}, f\left(u_{t}\right)\right]=a f\left(\left[u_{s}, u_{t}\right]\right)=0$. Since $y \in \operatorname{supp}\left(f\left(u_{t}\right)\right)$, it follows by Lemma 4.4 that $z \in \operatorname{st}_{2}(y)$. So, $\operatorname{lk}_{2}(x) \subset \operatorname{st}_{2}(y)$, that is, $x \prec_{N} y$. We show in the same way that, if $x \prec_{N} y$, then $s \prec_{M} t$.

Corollary 4.9 Let $s, t \in S$ and $x, y \in T$ such that $s \leftrightarrow_{f} x$ and $t \leftrightarrow_{f} y$. We have $s \equiv_{M} t$ if and only if $x \equiv_{N} y$.

Thanks to Corollary 4.9 we can define a map $f^{r}: S^{r} \rightarrow T^{r}$ as follows. Let $C \in S^{r}$. We choose $s \in C$ and $x \in T$ such that $s \leftrightarrow_{f} x$ and we set $f^{r}(C)=X$, where $X$ is the equivalence class of $x$ for the relation $\equiv_{N}$. It is clear that $f^{r}$ is invertible and $\left(f^{r}\right)^{-1}=\left(f^{-1}\right)^{r}$. Moreover, by Lemma 4.7, $m_{C, D}^{r}=n_{f^{r}(C), f^{r}(D)}^{r}$ for all $C, D \in S^{r}$, hence $f^{r}$ is an isomorphism from $M^{r}$ to $N^{r}$.

Lemma 4.10 Let $s \in S$ and $x, y \in T$ such that $s \leftrightarrow_{f} x$ and $y \in \operatorname{supp}\left(f\left(u_{s}\right)\right)$. Then $x \prec_{N} y$.

Proof Let $z \in \mathrm{lk}_{2}(x)$. We have $n_{x, z}=2$ and $s \in \operatorname{supp}\left(f^{-1}\left(u_{x}\right)\right)$, hence, by Lemma 4.5, $\left[u_{z}, f\left(u_{s}\right)\right]=0$. Since $y \in \operatorname{supp}\left(f\left(u_{s}\right)\right)$, it follows by Lemma 4.4 that $y \in \operatorname{st}_{2}(z)$, hence $z \in \operatorname{st}_{2}(y)$. This shows that $\mathrm{lk}_{2}(x) \subset \mathrm{st}_{2}(y)$, that is, $x \prec_{N} y$.

Lemma 4.11 Let $C \in S^{r}$. Then $\left|f^{r}(C)\right|=|C|$.

Proof Let $C \in S^{r}, X=f^{r}(C), s \in C$ and $x \in X$. Set $U=\left\{t \in S \mid s \prec_{M} t\right\}, U_{0}=\{t \in$ $S \mid s \prec_{M} t$ and $\left.s \not_{M} t\right\}, V=\left\{y \in T \mid x \prec_{N} y\right\}, V_{0}=\left\{y \in T \mid x \prec_{N} y\right.$ and $\left.x \not \equiv_{N} y\right\}$. If $E$ is a subset of $S$ (resp. of $T$ ) we denote by $\langle E\rangle$ the linear subspace of $L_{\mathbb{K}, 1}[M]$ (resp. of $\left.L_{\mathbb{K}, 1}[N]\right)$ spanned by $\left\{u_{t} \mid t \in E\right\}$. We have $C=U \backslash U_{0}$, hence $\operatorname{dim}(\langle U\rangle)-\operatorname{dim}\left(\left\langle U_{0}\right\rangle\right)=|C|$. Similarly, $\operatorname{dim}(\langle V\rangle)-\operatorname{dim}\left(\left\langle V_{0}\right\rangle\right)=|X|$. By Lemma 4.8 and Lemma 4.10 we have $f(\langle U\rangle) \subset\langle V\rangle$ and $f\left(\left\langle U_{0}\right\rangle\right) \subset\left\langle V_{0}\right\rangle$. Similarly, $f^{-1}(\langle V\rangle) \subset\langle U\rangle$ and $f^{-1}\left(\left\langle V_{0}\right\rangle\right) \subset\left\langle U_{0}\right\rangle$, hence $f(\langle U\rangle)=\langle V\rangle$ and $f\left(\left\langle U_{0}\right\rangle\right)=\left\langle V_{0}\right\rangle$. So, $|C|=\operatorname{dim}(\langle U\rangle)-\operatorname{dim}\left(\left\langle U_{0}\right\rangle\right)=\operatorname{dim}(\langle V\rangle)-\operatorname{dim}\left(\left\langle V_{0}\right\rangle\right)=|X|$.

Lemma 4.12 Let $C \in S^{r}$. Then $\operatorname{lb}\left(f^{r}(C)\right)=\operatorname{lb}(C)$.

Proof Let $C \in S^{r}$ and let $X=f^{r}(C)$. Note that, by Lemma 4.11, $|C|=|X|$. If $|C|=|X|=1$, then $\mathrm{lb}(C)=\mathrm{lb}(X)=0$. So, we can assume that $|C|=|X| \geq 2$. Suppose that there exists $x \in X$ such that $s \leftrightarrow_{f} x$ for all $s \in C$. Let $y \in X, y \neq x$. By Lemma 4.6 there exists $t \in S$ such that $t \leftrightarrow_{f} y$, and, by Corollary 4.9, we have $t \in C$. Choose $s \in C, s \neq t$. Then $s \neq t, x \neq y, s \leftrightarrow_{f} x$ and $t \leftrightarrow_{f} y$. Suppose that there is no $x \in X$ such that $s \leftrightarrow_{f} x$ for all $s \in C$. Then, obviously, there exist $s, t \in C, s \neq t$, and $x, y \in X, x \neq y$, such that $s \leftrightarrow_{f} x$ and $t \leftrightarrow_{f} y$. So, there always exist $s, t \in C, s \neq t$, and $x, y \in X$, $x \neq y$, such that $s \leftrightarrow_{f} x$ and $t \leftrightarrow_{f} y$. Then, by Lemma 4.7, $\operatorname{lb}(C)=m_{s, t}=n_{x, y}=\operatorname{lb}(X)$.

Proof of Theorem 4.1 Let $f: L_{\mathbb{K}}[M] \rightarrow L_{\mathbb{K}}[N]$ be an isomorphism. Then, by the above, $f$ induces an isomorphism $f^{r}: M^{r} \rightarrow N^{r}$ satisfying $\left|f^{r}(C)\right|=|C|$ and $\operatorname{lb}\left(f^{r}(C)\right)=\operatorname{lb}(C)$ for all $C \in S^{r}$. We conclude by Proposition 3.4 that $M$ and $N$ are isomorphic. 


\section{$5 \quad$ Rigidity of $\mathcal{A}[\mathcal{E}(c, d)]$}

Recall that, for $c, d \in \mathbb{N}$ such that $c \geq 1, d \geq 2$ and $\operatorname{gcd}(c, d)=1$, we denote by $\mathcal{E}(c, d)$ the set of even Coxeter matrices $M=\left(m_{s, t}\right)_{s, t \in S}$ such that $m_{s, t} \in\{2 c, \infty\} \cup\left\{2 d^{r} \mid r \geq 1\right\}$ for all $s, t \in S, s \neq t$. Our aim in the present section is to prove that the family $\mathcal{A}[\mathcal{E}(c, d)]$ is rigid (see Theorem 5.12 and Corollary 5.13). We first prove the case $c=1$ (see Theorem 5.1 and Corollary 5.2).

Theorem 5.1 Let $d \in \mathbb{N}, d \geq 2$, and $M, N \in \mathcal{E}(1, d)$. If $L[M]$ is isomorphic to $L[N]$, then $M$ is isomorphic to $N$.

Corollary 5.2 Let $d \in \mathbb{N}, d \geq 2$. Then $\mathcal{A}[\mathcal{E}(1, d)]$ is rigid.

Proof Let $M, N \in \mathcal{E}(1, d)$. If $A[M]$ is isomorphic to $A[N]$, then $\mathcal{T} \mathcal{G} r(A[M])$ is isomorphic to $\mathcal{T G} r(A[N])$. Since, by Theorem 2.1, $\mathcal{T} \mathcal{G} r(A[M])$ is isomorphic to $L[M]$ and $\mathcal{T} \mathcal{G} r(A[N])$ is isomorphic to $L[N]$, we conclude by Theorem 5.1 that $M$ is isomorphic to $N$.

We turn now to prove Theorem 5.1. So, we take two Coxeter matrices $M=\left(m_{s, t}\right)_{s, t \in S}$ and $N=$ $\left(n_{x, y}\right)_{x, y \in T}$ lying in $\mathcal{E}(1, d)$ and an isomorphism $f: L[M] \rightarrow L[N]$. Our aim is to prove that $M$ and $N$ are isomorphic.

We fix a prime number $p$ which divides $d$. The $p$-support of an element $\alpha=\sum_{s \in S} a_{s} u_{s} \in L_{1}[M]$ is defined to be $\operatorname{supp}_{p}(\alpha)=\left\{s \in S \mid \operatorname{gcd}\left(p, a_{s}\right)=1\right\}$. For $s \in S$ and $x \in T$ we set $s \leftrightarrow_{p, f} x$ if $s \in \operatorname{supp}_{p}\left(f^{-1}\left(u_{x}\right)\right)$ and $x \in \operatorname{supp}_{p}\left(f\left(u_{s}\right)\right)$.

Lemma 5.3 Let $s \in S$. There exists $x \in T$ such that $s \leftrightarrow_{p, f} x$.

Proof We argue like in the proof of Lemma 4.6. For $s \in S$ we set $f\left(u_{s}\right)=\sum_{x \in T} a_{s, x} u_{x}$, and for $x \in T$ we set $f^{-1}\left(u_{x}\right)=\sum_{s \in S} b_{x, s} u_{s}$. Let $s \in S$. Then

$$
u_{s}=\left(f^{-1} \circ f\right)\left(u_{S}\right)=\sum_{x \in T} \sum_{t \in S} a_{s, x} b_{x, t} u_{t},
$$

hence there exists $x \in T$ such that $\operatorname{gcd}\left(p, a_{s, x}\right)=1$ and $\operatorname{gcd}\left(p, b_{x, s}\right)=1$. Then $s \in \operatorname{supp}_{p}\left(f^{-1}\left(u_{x}\right)\right)$ and $x \in \operatorname{supp}_{p}\left(f\left(u_{s}\right)\right)$, that is, $s \leftrightarrow_{p, f} x$.

We set $L_{1}^{(d)}[M]=L_{1}[M], L_{2}^{(d)}[M]=d L_{2}[M]$, and $L^{(d)}[M]=L_{1}^{(d)}[M] \oplus L_{2}^{(d)}[M]$, and we define the Lie bracket $[\cdot, \cdot]^{(d)}$ of $L^{(d)}[M]$ as follows. For $\alpha, \beta \in L_{1}^{(d)}[M]$ we set $[\alpha, \beta]^{(d)}=d[\alpha, \beta]$, where $[\cdot, \cdot]$ denotes the Lie bracket of $L[M]$. On the other hand, we set $[\alpha, \beta]^{(d)}=0$ for all $(\alpha, \beta) \in$ $L_{2}^{(d)}[M] \times L^{(d)}[M]$. We define the Lie algebra $L^{(d)}[N]$ in the same way. It is easily seen that $f$ induces an isomorphism $f^{(d)}: L^{(d)}[M] \rightarrow L^{(d)}[N]$.

Let $M^{(d)}=\left(m_{s, t}^{(d)}\right)_{s, t \in S}$ be the Coxeter matrix defined as follows.

- $m_{s, t}^{(d)}=2$ if $m_{s, t} \in\{2,2 d\}$.

- Let $r \in \mathbb{N}, r \geq 2$. Then $m_{s, t}^{(d)}=2 d^{r-1}$ if $m_{s, t}=2 d^{r}$. 
- $m_{s, t}^{(d)}=\infty$ if $m_{s, t}=\infty$.

We define $N^{(d)}$ in the same way. Note that $M^{(d)}$ and $N^{(d)}$ are even Coxeter matrices lying in $\mathcal{E}(1, d)$. The proof of the following lemma is left to the reader.

Lemma 5.4 (1) We have $L^{(d)}[M] \simeq L\left[M^{(d)}\right]$ and $L^{(d)}[N] \simeq L\left[N^{(d)}\right]$.

(2) Let $s \in S$ and $x \in T$. We have $s \leftrightarrow_{p, f} x$ if and only if $s \leftrightarrow_{p, f^{(d)}} x$.

We define a right-angled Coxeter matrix $M_{(p)}=\left(m_{(p), s, t}\right)_{s, t \in S}$ as follows.

- $m_{(p), s, t}=2$ if $m_{s, t}=2$.

- $m_{(p), s, t}=\infty$ if $m_{s, t} \in\left\{2 d^{r} \mid r \in \mathbb{N}, r \geq 1\right\} \cup\{\infty\}$.

We define the right-angled Coxeter matrix $N_{(p)}$ in the same way. Let $\mathbb{F}_{p}=\mathbb{Z} / p \mathbb{Z}$, and let $f_{(p)}$ : $\mathbb{F}_{p} \otimes L[M] \rightarrow \mathbb{F}_{p} \otimes L[N]$ be the isomorphism induced by $f$. Again, the proof of the following is left to the reader.

Lemma 5.5 (1) We have $\mathbb{F}_{p} \otimes L[M] \simeq L_{\mathbb{F}_{p}}\left[M_{(p)}\right]$ and $\mathbb{F}_{p} \otimes L[N] \simeq L_{\mathbb{F}_{p}}\left[N_{(p)}\right]$.

(2) Let $s \in S$ and $x \in T$. We have $s \leftrightarrow_{p, f} x$ if and only if $s \leftrightarrow_{(p)} x$.

Lemma 5.6 Let $s, t \in S, s \neq t$, and $x, y \in T, x \neq y$, such that $s \leftrightarrow_{p, f} x$ and $t \leftrightarrow_{p, f} y$. Then $m_{s, t}=n_{x, y}$.

Proof Let $\rho \in \mathbb{N}$ such that $E_{2 d^{\rho}}(M) \neq \emptyset$ or $E_{2 d^{\rho}}(N) \neq \emptyset$, and $E_{2 d^{k}}(M)=E_{2 d^{k}}(N)=\emptyset$ for all $k>\rho$ (we set $\rho=0$ if $m_{s, t}=\infty$ for all $s, t \in S, s \neq t$, and $n_{x, y}=\infty$ for all $x, y \in T, x \neq y$ ). We argue by induction on $\rho$. If $\rho=0$, then $M$ and $N$ are both right-angled Coxeter matrices and the result follows from Lemma 4.7. So, we can assume that $\rho \geq 1$ and that the inductive hypothesis holds. Suppose that $m_{s, t}=2$. Then $m_{(p), s, t}=2$ and, by Lemma 5.5, $s \leftrightarrow_{f_{(p)}} x$ and $t \leftrightarrow f_{(p)} y$, hence, by Lemma 4.7, $n_{(p), x, y}=2$, and therefore $n_{x, y}=2$. We show in the same way that, if $n_{x, y}=2$, then $m_{s, t}=2$. So, we can assume that $m_{s, t} \geq 2 d$ and $n_{x, y} \geq 2 d$. By Lemma 5.4, $s \leftrightarrow_{p, f^{(d)}} x$ and $t \leftrightarrow_{p, f^{(d)}} y$, hence, by the inductive hypothesis, $m_{s, t}^{(d)}=n_{x, y}^{(d)}$. If $m_{s, t}^{(d)}=n_{x, y}^{(d)}=\infty$, then $m_{s, t}=n_{x, y}=\infty$. If $m_{s, t}^{(d)}=n_{x, y}^{(d)}<\infty$, then $m_{s, t}=n_{x, y}=d m_{s, t}^{(d)}=d n_{x, y}^{(d)}$, since $m_{s, t} \geq 2 d$ and $n_{x, y} \geq 2 d$.

Lemma 5.7 Let $s, t \in S$ and $x, y \in T$ such that $s \leftrightarrow_{p, f} x$ and $t \leftrightarrow_{p, f} y$. We have $s \prec_{M} t$ if and only if $x \prec_{N} y$.

Proof Let $\rho \in \mathbb{N}$ such that $E_{2 d^{\rho}}(M) \neq \emptyset$ or $E_{2 d^{\rho}}(N) \neq \emptyset$, and $E_{2 d^{k}}(M)=E_{2 d^{k}}(N)=\emptyset$ for all $k>\rho$ (again, we set $\rho=0$ if $m_{s, t}=\infty$ for all $s, t \in S, s \neq t$, and $n_{x, y}=\infty$ for all $x, y \in T, x \neq y$ ). We argue by induction on $\rho$. Assume that $\rho=0$, that is, $M$ and $N$ are both right-angled Coxeter matrices. Then $f$ induces an isomorphism $f_{\mathbb{F}_{p}}: L_{\mathbb{F}_{p}}[M]=\mathbb{F}_{p} \otimes L[M] \rightarrow \mathbb{F}_{p} \otimes L[N]=L_{\mathbb{F}_{p}}[N]$, hence, by Lemma 4.8, we have $s \prec_{M} t$ if and only if $x \prec_{N} y$.

Now, assume that $\rho \geq 1$ and that the inductive hypothesis holds. Suppose that $s \prec_{M} t$. We take $m \in \mathbb{N}$, $m \geq 2$, and we prove that $\mathrm{lk}_{m}(x) \subset \mathrm{st}_{m}(y)$. This will prove that $x \prec_{N} y$. Since $M, N \in \mathcal{E}(1, d)$, we can assume that $m \in\left\{2 d^{k} \mid 0 \leq k \leq \rho\right\}$. 
Suppose that $m=2$. For $r \in S$ we denote by $\operatorname{lk}_{(p), 2}(r)$ the 2 -link of $r$ and by $\operatorname{st}_{(p), 2}(r)$ the 2 -star of $r$ with respect to $M_{(p)}$. Similarly, for $z \in T$ we denote by $\operatorname{lk}_{(p), 2}(z)$ the 2 -link of $z$ and by $\operatorname{st}_{(p), 2}(z)$ the 2-star of $z$ with respect to $N_{(p)}$. Observe that $\operatorname{lk}_{2}(s)=\mathrm{lk}_{(p), 2}(s)$ and $\mathrm{st}_{2}(t)=\mathrm{st}_{(p), 2}(t)$, hence $\mathrm{lk}_{(p), 2}(s) \subset \operatorname{st}_{(p), 2}(t)$, that is, $s \prec_{M_{(p)}} t$. Moreover, by Lemma 5.5, $s \leftrightarrow_{f_{(p)}} x$ and $t \leftrightarrow_{f_{(p)}} y$, hence, by Lemma 4.8, $x \prec_{N_{(p)}} y$. This means that $\operatorname{lk}_{2}(x)=\operatorname{lk}_{(p), 2}(x) \subset \operatorname{st}_{(p), 2}(y)=\operatorname{st}_{2}(y)$.

Assume that $m \geq 2 d$, that is, $m=2 d^{k+1}$ for some $k \in\{0, \ldots, \rho-1\}$. For $r \in S$ and $0 \leq k \leq \rho-1$ we denote by $\mathrm{lk}_{2 d^{k}}^{(d)}(r)$ the $2 d^{k}$-link of $r$ and by st ${ }_{2 d^{k}}^{(d)}(r)$ the $2 d^{k}$-star of $r$ with respect to $M^{(d)}$. Similarly, for $z \in T$ and $0 \leq k \leq \rho-1$ we denote by $\operatorname{lk}_{2 d^{k}}^{(d)}(z)$ the $2 d^{k}$-link of $z$ and by $\operatorname{st}_{2 d^{k}}^{(d)}(z)$ the $2 d^{k}$-star of $z$ with respect to $N^{(d)}$. Observe that $\mathrm{lk}_{2 d^{k+1}}(s)=\mathrm{lk}_{2 d^{k}}^{(d)}(s)$ and $\mathrm{st}_{2 d^{k+1}}(t)=\mathrm{st}_{2 d^{k}}^{(d)}(t)$ for all $0 \leq k \leq \rho-1$, hence $\mathrm{lk}_{2 d^{k}}^{(d)}(s) \subset \mathrm{st}_{2 d^{k}}^{(d)}(t)$ for all $0 \leq k \leq \rho-1$, and therefore $s \prec_{M^{(d)}} t$. Moreover, by Lemma 5.4, $s \leftrightarrow_{p, f^{(d)}} x$ and $t \leftrightarrow_{p, f^{(d)}} y$, hence, by the inductive hypothesis, $x \prec_{N^{(d)}} y$. This implies that $\mathrm{lk}_{2 d^{k+1}}(x)=\mathrm{lk}_{2 d^{k}}^{(d)}(x) \subset \mathrm{st}_{2 d^{k}}^{(d)}(y)=\mathrm{st}_{2 d^{k+1}}(y)$ for all $0 \leq k \leq \rho-1$. So, $\mathrm{lk}_{m}(x) \subset \operatorname{st}_{m}(y)$.

Corollary 5.8 Let $s, t \in S$ and $x, y \in T$ such that $s \leftrightarrow_{p, f} x$ and $t \leftrightarrow_{p, f} y$. We have $s \equiv_{M} t$ if and only if $x \equiv_{N} y$.

Thanks to Corollary 5.8 we can define a map $f^{r}: S^{r} \rightarrow T^{r}$ as follows. Let $C \in S^{r}$. We choose $s \in C$ and $x \in T$ such that $s \leftrightarrow_{p, f} x$ and we set $f^{r}(C)=X$, where $X$ is the equivalence class of $x$ for the relation $\equiv_{N}$. It is clear that $f^{r}$ is invertible and $\left(f^{r}\right)^{-1}=\left(f^{-1}\right)^{r}$. Moreover, by Lemma 5.6, $m_{C, D}^{r}=n_{f^{r}(C), f^{r}(D)}^{r}$ for all $C, D \in S^{r}$, hence $f^{r}$ determines an isomorphism from $M^{r}$ to $N^{r}$.

Lemma 5.9 Let $s \in S$ and $x, y \in T$ such that $s \leftrightarrow_{p, f} x$ and $y \in \operatorname{supp}_{p}\left(f\left(u_{s}\right)\right)$. Then $x \prec_{N} y$.

Proof Let $\rho \in \mathbb{N}$ such that $E_{2 d^{\rho}}(M) \neq \emptyset$ or $E_{2 d^{\rho}}(N) \neq \emptyset$, and $E_{2 d^{k}}(M)=E_{2 d^{k}}(N)=\emptyset$ for all $k>\rho$ (and, as ever, $\rho=0$ if $m_{s, t}=\infty$ for all $s, t \in S, s \neq t$, and $n_{x, y}=\infty$ for all $x, y \in T, x \neq y$ ). We argue by induction on $\rho$. Suppose that $\rho=0$, that is, $M$ and $N$ are both right-angled Coxeter matrices. Then $f$ induces an isomorphism $f_{(p)}: L_{\mathbb{F}_{p}}[M]=\mathbb{F}_{p} \otimes L[M] \rightarrow \mathbb{F}_{p} \otimes L[N]=L_{\mathbb{F}_{p}}[N]$ hence, by Lemma $4.10, x \prec_{N} y$.

Now, we assume that $\rho \geq 1$ and that the inductive hypothesis holds. We take $m \in \mathbb{N}, m \geq 2$, and we show that $\mathrm{lk}_{m}(x) \subset \operatorname{st}_{m}(y)$. This will show that $x \prec_{N} y$. Again, since $M$ and $N$ lie in $\mathcal{E}(1, d)$, we can suppose that $m \in\left\{2 d^{k} \mid 0 \leq k \leq \rho\right\}$.

Suppose first that $m=2$. For $z \in T$ we denote by $\operatorname{lk}_{(p), 2}(z)$ the 2 -link of $z$ and by $\operatorname{st}_{(p), 2}(z)$ the 2 -star of $z$ with respect to $N_{(p)}$. By Lemma 5.5, $s \leftrightarrow_{f_{(p)}} x$ hence, by Lemma 4.10, $x \prec_{N_{(p)}} y$. This implies that $\mathrm{lk}_{2}(x)=\mathrm{lk}_{(p), 2}(x) \subset \operatorname{st}_{(p), 2}(y)=\mathrm{st}_{2}(y)$.

Now, suppose that $m \geq 2 d$, that is, $m=2 d^{k+1}$ for some $k \in\{0, \ldots, \rho-1\}$. For $z \in T$ and $0 \leq k \leq \rho-1$ we denote by $\operatorname{lk}_{2 d^{k}}^{(d)}(z)$ the $2 d^{k}$-link of $z$ and by $\operatorname{st}_{2 d^{k}}^{(d)}(z)$ the $2 d^{k}$-star of $z$ with respect to $N^{(d)}$. By Lemma 5.4, $s \leftrightarrow_{p, f^{(d)}} x$ hence, by the inductive hypothesis, $x \prec_{N^{(d)}} y$. This implies that $\mathrm{lk}_{2 d^{k+1}}(x)=\mathrm{lk}_{2 d^{k}}^{(d)}(x) \subset \mathrm{st}_{2 d^{k}}^{(d)}(y)=\mathrm{st}_{2 d^{k+1}}(y)$ for all $0 \leq k \leq \rho-1$. So, $\mathrm{lk}_{m}(x) \subset \operatorname{st}_{m}(y)$.

Lemma 5.10 Let $C \in S^{r}$. Then $\left|f^{r}(C)\right|=|C|$. 
Proof The proof is almost identical to the one of Lemma 4.11. Let $C \in S^{r}, X=f^{r}(C), s \in C$ and $x \in X$. Set $U=\left\{t \in S \mid s \prec_{M} t\right\}, U_{0}=\left\{t \in S \mid s \prec_{M} t\right.$ and $\left.s \not_{M} t\right\}, V=\left\{y \in T \mid x \prec_{N} y\right\}$, $V_{0}=\left\{y \in T \mid x \prec_{N} y\right.$ and $\left.x \not_{N} y\right\}$. If $E$ is a subset of $S$ (resp. of $T$ ) we denote by $\langle E\rangle$ the $\mathbb{Z}$-submodule of $L_{1}[M]$ (resp. of $L_{1}[N]$ ) spanned by $\left\{u_{t} \mid t \in E\right\}$. We have $C=U \backslash U_{0}$, hence $\operatorname{rk}(\langle U\rangle)-\operatorname{rk}\left(\left\langle U_{0}\right\rangle\right)=|C|$. Similarly, $\operatorname{rk}(\langle V\rangle)-\operatorname{rk}\left(\left\langle V_{0}\right\rangle\right)=|X|$. On the other hand, by Lemma 5.7 and Lemma 5.9, $f(\langle U\rangle) \subset\langle V\rangle$ and $f\left(\left\langle U_{0}\right\rangle\right) \subset\left\langle V_{0}\right\rangle$. Similarly, $f^{-1}(\langle V\rangle) \subset\langle U\rangle$ and $f^{-1}\left(\left\langle V_{0}\right\rangle\right) \subset\left\langle U_{0}\right\rangle$, hence $f(\langle U\rangle)=\langle V\rangle$ and $f\left(\left\langle U_{0}\right\rangle\right)=\left\langle V_{0}\right\rangle$. So, $|C|=\operatorname{rk}(\langle U\rangle)-\operatorname{rk}\left(\left\langle U_{0}\right\rangle\right)=\operatorname{rk}(\langle V\rangle)-\operatorname{rk}\left(\left\langle V_{0}\right\rangle\right)=$ $|X|$.

Lemma 5.11 Let $C \in S^{r}$. Then $\operatorname{lb}\left(f^{r}(C)\right)=\operatorname{lb}(C)$.

Proof We argue in the same way as in the proof of Lemma 4.12. Let $C \in S^{r}$, and let $X=f^{r}(C)$. Note that, by Lemma 5.10, $|C|=|X|$. If $|C|=|X|=1$, then $\operatorname{lb}(C)=\operatorname{lb}(X)=0$. So, we can assume that $|C|=|X| \geq 2$. Suppose there exists $x \in X$ such that $s \leftrightarrow_{p, f} x$ for all $s \in C$. Let $y \in X, y \neq x$. By Lemma 5.3 there exists $t \in S$ such that $t \leftrightarrow_{p, f} y$ and, by Corollary 5.8, $t \in C$. Take $s \in C$ such that $s \neq t$. Then $s \neq t, x \neq y, s \leftrightarrow_{p, f} x$ and $t \leftrightarrow_{p, f} y$. If there is no $x \in X$ such that $s \leftrightarrow_{p, f} x$ for all $s \in C$, then, obviously, there exist $s, t \in C, s \neq t$, and $x, y \in X, x \neq y$, such that $s \leftrightarrow_{p, f} x$ and $t \leftrightarrow_{p, f} y$. So, there always exist $s, t \in C, s \neq t$, and $x, y \in X, x \neq y$, such that $s \leftrightarrow_{p, f} x$ and $t \leftrightarrow_{p, f} y$. Then, by Lemma 5.6, $\operatorname{lb}(C)=m_{s, t}=n_{x, y}=\mathrm{lb}(X)$.

Proof of Theorem 5.1 Let $f: L[M] \rightarrow L[N]$ be an isomorphism. Then, by the above, there exists an isomorphism $f^{r}: M^{r} \rightarrow N^{r}$ such that $\left|f^{r}(C)\right|=|C|$ and $\operatorname{lb}\left(f^{r}(C)\right)=\operatorname{lb}(C)$ for all $C \in S^{r}$. We conclude by Proposition 3.4 that $M$ and $N$ are isomorphic.

We turn now to the general case.

Theorem 5.12 Let $c, d \in \mathbb{N}$ such that $c \geq 1, d \geq 2$ and $\operatorname{gcd}(c, d)=1$, and let $M, N \in \mathcal{E}(c, d)$. If $L[M]$ is isomorphic to $L[N]$, then $M$ is isomorphic to $N$.

The following corollary can be proved from Theorem 5.12 in the same way as Corollary 5.2 is proved from Theorem 5.1.

Corollary 5.13 Let $c, d \in \mathbb{N}$ such that $c \geq 1, d \geq 2$ and $\operatorname{gcd}(c, d)=1$. Then $\mathcal{A}[\mathcal{E}(c, d)]$ is rigid.

Proof of Theorem 5.12 Let $f: L[M] \rightarrow L[N]$ be an isomorphism. We define a graded Lie $\mathbb{Z}$-algebra $L^{(c)}[M]$ as follows. We set $L_{1}^{(c)}[M]=L_{1}[M], L_{2}^{(c)}[M]=c L_{2}[M]$, and $L^{(c)}[M]=L_{1}^{(c)}[M] \oplus L_{2}^{(c)}[M]$. We define the Lie bracket $[\cdot, \cdot]^{(c)}$ of $L^{(c)}[M]$ as follows. For $\alpha, \beta \in L_{1}^{(c)}[M]$ we set $[\alpha, \beta]^{(c)}=c[\alpha, \beta]$, where $[\cdot, \cdot]$ denotes the Lie bracket of $L[M]$. On the other hand, we set $[\alpha, \beta]^{(c)}=0$ for all $(\alpha, \beta) \in$ $L_{2}^{(c)}[M] \times L^{(c)}[M]$. We define $L^{(c)}[N]$ in the same way. It is easily seen that $f$ induces an isomorphism $f^{(c)}: L^{(c)}[M] \rightarrow L^{(c)}[N]$.

Let $M^{(c)}=\left(m_{s, t}^{(c)}\right)_{s, t \in S}$ be the Coxeter matrix defined as follows.

- $m_{s, t}^{(c)}=2$ if $m_{s, t}=2 c$. 
- $m_{s, t}^{(c)}=m_{s, t}$ if $m_{s, t} \neq 2 c$.

We define $N^{(c)}$ in the same way. It is clear that $M^{(c)}$ and $N^{(c)}$ are both even Coxeter matrices lying in $\mathcal{E}(1, d)$, that $M$ is isomorphic to $N$ if and only if $M^{(c)}$ is isomorphic to $N^{(c)}$, that $L^{(c)}[M] \simeq L\left[M^{(c)}\right]$, and that $L^{(c)}[N] \simeq L\left[N^{(c)}\right]$. By Theorem 5.1, $M^{(c)}$ and $N^{(c)}$ are isomorphic, hence $M$ and $N$ are isomorphic.

\section{An example}

Consider the following even Coxeter matrices.

$$
M_{0}=\left(\begin{array}{ccc}
1 & 6 & 2 \\
6 & 1 & 10 \\
2 & 10 & 1
\end{array}\right), N_{0}=\left(\begin{array}{ccc}
1 & 2 & 2 \\
2 & 1 & 30 \\
2 & 30 & 1
\end{array}\right)
$$

It is obvious that $M_{0}$ and $N_{0}$ are not isomorphic. Furthermore, we also have the following.

Lemma 6.1 The Artin groups $A\left[M_{0}\right]$ and $A\left[N_{0}\right]$ are not isomorphic.

Proof The group $A\left[M_{0}\right]$ is a two-dimensional Artin group in the sense of Charney-Davis [8], hence, by Charney-Davis [8] [9, Corollary 1.4.2], the cohomological dimension of $A\left[M_{0}\right]$ is 2 . On the other hand, $A\left[N_{0}\right]$ is an Artin group of spherical type of rank 3, hence, by Charney-Davis [9, Corollary 1.4.2], the cohomological dimension of $A\left[N_{0}\right]$ is 3. So, $A\left[M_{0}\right]$ and $A\left[N_{0}\right]$ are not isomorphic.

However, we have the following.

Proposition 6.2 The Lie algebras $L\left[M_{0}\right]$ and $L\left[N_{0}\right]$ are isomorphic.

Proof We denote by $\mathcal{U}=\left\{u_{1}, u_{2}, u_{3}\right\}$ the standard $\mathbb{Z}$-basis of $L_{1}\left[M_{0}\right]$ and by $\mathcal{U}^{\prime}=\left\{u_{1}^{\prime}, u_{2}^{\prime}, u_{3}^{\prime}\right\}$ the standard $\mathbb{Z}$-basis of $L_{1}\left[N_{0}\right]$. On the other hand, we denote by $\mathcal{V}=\left\{v_{1,2}, v_{2,3}\right\}$ the standard generating set of $L_{2}\left[M_{0}\right]$ and by $\mathcal{V}^{\prime}=\left\{v_{2,3}^{\prime}\right\}$ the standard generating set of $L_{2}\left[N_{0}\right]$. Let $f_{1}: L_{1}\left[M_{0}\right] \rightarrow L_{1}\left[N_{0}\right]$ and $g_{1}: L_{1}\left[N_{0}\right] \rightarrow L_{1}\left[M_{0}\right]$ be the $\mathbb{Z}$-linear maps whose matrices relative to the basis $\mathcal{U}$ and $\mathcal{U}^{\prime}$ are $F$ and $G$, respectively, where

$$
F=\left(\begin{array}{lll}
2 & 0 & 1 \\
0 & 1 & 0 \\
5 & 0 & 3
\end{array}\right), G=\left(\begin{array}{ccc}
3 & 0 & -1 \\
0 & 1 & 0 \\
-5 & 0 & 2
\end{array}\right) .
$$

A direct calculation shows that $f_{1} \circ g_{1}=\mathrm{id}$ and $g_{1} \circ f_{1}=\mathrm{id}$, hence $f_{1}$ is an isomorphism of $\mathbb{Z}$-modules and $g_{1}$ is the inverse of $f_{1}$. We define $f_{2}: L_{2}\left[M_{0}\right] \rightarrow L_{2}\left[N_{0}\right]$ by $f_{2}\left(v_{1,2}\right)=-5 v_{2,3}^{\prime}$ and $f_{2}\left(v_{2,3}\right)=3 v_{2,3}^{\prime}$. It is easily checked that $f_{2}$ is well-defined, it is invertible, and its inverse $g_{2}: L_{2}\left[N_{0}\right] \rightarrow L_{2}\left[M_{0}\right]$ is defined by $g_{2}\left(v_{2,3}^{\prime}\right)=v_{1,2}+2 v_{2,3}$. We check with a direct calculation that $\left[f_{1}\left(u_{i}\right), f_{1}\left(u_{j}\right)\right]=f_{2}\left(\left[u_{i}, u_{j}\right]\right)$ for all $i, j \in\{1,2,3\}$, hence $f_{1}$ and $f_{2}$ induce an isomorphism $f: L\left[M_{0}\right] \rightarrow L\left[N_{0}\right]$ of Lie $\mathbb{Z}$-algebras. 


\section{References}

[1] P Bahls, The isomorphism problem in Coxeter groups, Imperial College Press, London, 2005.

[2] H Bass, A Lubotzky, Linear-central filtrations on groups, The mathematical legacy of Wilhelm Magnus: groups, geometry and special functions (Brooklyn, NY, 1992), 45-98, Contemp. Math., 169, Amer. Math. Soc., Providence, RI, 1994.

[3] R Blasco-García, J I Cogolludo-Agustín, Quasi-projectivity of even Artin groups, Geom. Topol. 22 (2018), no. 7, 3979-4011.

[4] R Blasco-García, A Juhász, L Paris, Note on the residual finiteness of Artin groups, J. Group Theory 21 (2018), no. 3, 531-537.

[5] R Blasco-García, C Martínez-Pérez, L Paris, Poly-freeness of even Artin groups of FC type, Groups Geom. Dyn. 13 (2019), no. 1, 309-325.

[6] N Brady, J P McCammond, B Mühlherr, W D Neumann, Rigidity of Coxeter groups and Artin groups, Proceedings of the Conference on Geometric and Combinatorial Group Theory, Part I (Haifa, 2000), Geom. Dedicata 94 (2002), 91-109.

[7] R Charney, The Tits conjecture for locally reducible Artin groups, Internat. J. Algebra Comput. 10 (2000), no. 6, 783-797.

[8] R Charney, M W Davis, The $K(\pi, 1)$-problem for hyperplane complements associated to infinite reflection groups, J. Amer. Math. Soc. 8 (1995), no. 3, 597-627.

[9] R Charney, M W Davis, Finite $K(\pi, 1)$ s for Artin groups, Prospects in topology (Princeton, NJ, 1994), 110-124, Ann. of Math. Stud., 138, Princeton Univ. Press, Princeton, NJ, 1995.

[10] C Droms, Isomorphisms of graph groups, Proc. Amer. Math. Soc. 100 (1987), no. 3, 407-408.

[11] K H Kim, L Makar-Limanov, J Neggers, F W Roush, Graph algebras, J. Algebra 64 (1980), no. 1, 46-51.

[12] T Koberda, Right-angled Artin groups and a generalized isomorphism problem for finitely generated subgroups of mapping class groups, Geom. Funct. Anal. 22 (2012), no. 6, 1541-1590.

[13] M Lazard, Sur les groupes nilpotents et les anneaux de Lie, Ann. Sci. Ecole Norm. Sup. (3) 71 (1954), 101-190.

[14] W Magnus, A Karrass, D Solitar, Combinatorial group theory. Presentations of groups in terms of generators and relations, Reprint of the 1976 second edition, Dover Publications, Inc., Mineola, NY, 2004.

[15] L Paris, Artin groups of spherical type up to isomorphism, J. Algebra 281 (2004), no. 2, 666-678.

Rubén Blasco-García, Departamento de Matemáticas, IUMA, Universidad de Zaragoza, C. Pedro Cerbuna 12, 50009 Zaragoza, Spain

Luis Paris, IMB, UMR 5584, CNRS, Univ. Bourgogne Franche-Comté, 21000 Dijon, France

rubenb@unizar.es, lparis@u-bourgogne.fr 